\title{
ARTíCULO \\ Efecto del tamaño de malla en los valores de densidad y biomasa de la comunidad bentónica estuarina del Estero de Punta Banda, Baja California
}

\author{
Effect of sieve mesh size in the density and biomass values of the \\ estuarine benthic community from Estero \\ Punta Banda, Baja California \\ Juan Carlos Rubio-Polania ${ }^{1}$, Elena Solana-Arellano ${ }^{1}$, \\ Victoria Díaz-Castañeda ${ }^{1}$ y Olga Flores-Uzeta ${ }^{1}$
}

\begin{abstract}
'División de Oceanología, Laboratorio de Ecología Matemática, CICESE, Carretera Ensenada-Tijuana No. 3918, Zona Playitas, C.P. 22860, Ensenada, B.C., México. jcrubiopolania@ gmail.com

Abstract.- Retention of macroinvertebrates in sieves of sizes $1.0 \mathrm{~mm}$ and $0.5 \mathrm{~mm}$ were analyzed, with the aim of evaluating the effect of sieve size on density, biomass, and organisms' size in a benthic community from Estero de Punta Banda, Baja California, associated with a Zostera marina meadow. Data from 11 sampling dates from August 2009 to May 2011 were used to reach this objective. These were divided into two periods, the first between August 2009 to April 2010, and the second, between July 2010 to May 2011. A total of 13,606 organisms were collected distributed in 9 phyla and 10 classes. Nematodes showed the highest density values, meanwhile polychaetes retained in sieves of $1.0 \mathrm{~mm}$ showed the highest values of biomass. We found significant differences between density values of organisms retained in different sieves. These differences were attributed to a loss of 34 to $75 \%$ organisms due to the use of $1.0 \mathrm{~mm}$ sieve. There were no significant differences either in biomass values or in the size of the organisms in both periods, possibly due to the presence of $r$ species strategy across the period of study. Our results indicate that the $1.0 \mathrm{~mm}$ and $0.5 \mathrm{~mm}$ sieves are appropriate to evaluate the biomass and abundance of macrofauna communities in seagrass meadows areas of temperate regions, respectively. Possibly, the best sieve to use in places with no information about biodiversity patterns or seasonal fluctuation is the one of $0.5 \mathrm{~mm}$.
\end{abstract}

Key words: Benthos, mesh size sieve, Estero de Punta Banda, anti-estuary, Baja California

\begin{abstract}
Resumen.- La retención de macroinvertebrados en los tamices de 1,0 $\mathrm{mm}$ y 0,5 $\mathrm{mm}$ fue analizada, con el objetivo de evaluar el efecto del diámetro de malla sobre la densidad, biomasa y cambio en el tamaño de los organismos de una comunidad bentónica asociados a pastos marinos Zostera marina del Estero de Punta Banda, Baja California. Datos de 11 fechas de muestreo de agosto 2009 a mayo 2011 fueron usados para alcanzar este objetivo. Estos fueron divididos en 2 períodos, el primero entre agosto 2009 a abril 2010 y el segundo, entre junio de 2010 a mayo 2011. Un total de 13.606 organismos fueron recolectados, distribuidos en 9 phyla y 10 clases. Los Nematodos presentaron los valores más altos de densidad mientras los poliquetos retenidos en el tamiz de 1,0 $\mathrm{mm}$ presentaron los valores más elevados de biomasa. Se encontraron diferencias significativas entre los valores de densidad de los organismos retenidos en los tamices. Estas diferencias fueron atribuidas a una pérdida del 34 al $75 \%$ de los organismos debida al uso del tamiz de 1,0 mm. No hubo diferencias significativas en los valores de biomasa ni en el tamaño de los organismos en los dos periodos, posiblemente a causa de la presencia de especies de estrategia $r$ a lo largo del periodo de estudio. Los resultados indican que los tamices de $1,0 \mathrm{~mm}$ y $0,5 \mathrm{~mm}$ son apropiados para evaluar la biomasa y la densidad de comunidades macrofaunales en praderas de pastos marinos de áreas templadas, respectivamente. Es posible que el mejor tamiz para ser utilizado en lugares sin información sobre los patrones de biodiversidad y fluctuación estacional sea el tamiz de 0,5 mm.
\end{abstract}

Palabras clave: Bentos, diámetro de malla, Estero de Punta Banda, antiestuario, Baja California

\section{INTRODUCCIÓN}

Los ecosistemas antiestuarinos se caracterizan por presentar valores de temperatura y salinidad más altos en su parte interna que en su boca (Largier et al. 1997), siendo estos ecosistemas afectados en mayor medida por las condiciones presentes en la zona costera adyacente. Ante esta característica, Baja
California es una de las áreas a nivel mundial donde es posible encontrar dichos ecosistemas, en los cuales prosperan de manera conspicua diferentes comunidades macrofaunales marinas (Quiroz-Vázquez et al. 2005). 
La macrofauna bentónica de fondos blandos intermareales y submareales es definida como todos aquellos organismos que son retenidos en tamices que presentan un diámetro de malla de 0,5 mm (Holme \& McIntyre 1984). Las características intrínsecas de las comunidades macrofaunales (e.g., poca movilidad, alta sensibilidad y respuesta rápida ante fluctuaciones de las variables fisicoquímicas del medio), las han convertido en una herramienta ecológica importante debido a que son indicadores de diferentes condiciones ambientales, ya sean, naturales o antropogénicas (Pearson \& Rosenberg 1978, Pocklington \& Well 1992, Warwick 1993, Couto et al. 2010).

Es de vital importancia la descripción de las variaciones anuales de las comunidades bentónicas, debido a que esto permite detectar de manera clara potenciales disturbios antropogénicos (Whitlow \& Grabowski 2012). Para tal fin, es necesario determinar la presencia-ausencia, dominancia relativa y fluctuaciones estacionales de las especies presentes en un ecosistema (Rygg 1985, Abel 1996, Rosenberg et al. 2004); así como establecer los métodos adecuados para su valoración, debido a que las técnicas de muestreo y protocolos de recolección y procesamiento de las muestras influyen fuertemente los resultados (Tanaka \& Pereira 1998, Morin $e t$ al. 2004).

Un punto crítico es el tamaño de malla utilizado en la separación de organismos del sedimento, ya que entrega información sobre la frecuencia de organismos adultos y juveniles que integran la comunidad (Thompson et al. 2003, Lampadariou et al. 2005, Buss \& Borges 2008).

Aunque no existen pautas claras en el uso de tamices para la separación de los organismos macrofaunales, si existen tendencias en el uso de diámetros de malla que fluctúan entre 0,5 y 1,5 mm. Por ejemplo, Gray \& Elliott (2009), comentan el uso de tamices de 0,5 y 1,0 mm para estudios ambientales mientras que Eleftheriou \& McIntyre (2005), recomiendan tamices de 1,4 y 1,0 mm para sedimentos gruesos y 0,5 para sedimentos finos; lo cual conduce a cierta arbitrariedad en el uso de tamices.

Reish (1959), usando la densidad y la biomasa, demostró el efecto que tiene el diámetro de malla sobre dichas variables, sin llegar a recomendar el uso de un diámetro de malla en particular, a pesar de haber realizado su evaluación en las costas de California; donde es posible observar un patrón de reclutamiento unimodal donde se presenta la adición de organismos de pequeño tamaño a la comunidad debido a las variaciones de temperatura causadas por la estacionalidad (Botsford 2001).
A su vez Bachelet (1990) reporta fluctuaciones estacionales en la retención de organismos y resultados confiables usando un tamiz de 1,0 $\mathrm{mm}$ para valoraciones de biomasa; variable que presenta una propiedad conservativa en el patrón de distribución de sus valores en comunidades bentónicas de fondos blandos, donde organismos de gran tamaño con valores bajos de densidad relativa presentan los valores más altos de biomasa (Reish 1959, Schwinghamer 1981), Según RubioPolania (2013), esto se puede observar de manera detallada al usar un mayor número de tamices en la separación de los organismos, sin embargo pasa desapercibido en la mayoría de los estudios debido al uso tradicional de los tamices de 1,0 y $0,5 \mathrm{~mm}$.

Un efecto poco discutido en la mayoría de las evaluaciones de comunidades bentónicas es la fluctuación interanual en el tamaño de los organismos, lo cual puede darse como respuesta a variaciones en la disponibilidad de nutrientes o al efecto de fluctuaciones climáticas interanuales (e.g., El Niño Oscilación del Sur ENOS y la Oscilación Decadal del Pacifico ODP) (Sellanes et al. 2007), que afectan los valores de la salinidad y la temperatura, siendo estas variables importantes en el reclutamiento de especies al bentos ya que estas variables están correlacionadas positivamente con la cantidad de larvas, desarrollo y viabilidad de los organismos presentes en una comunidad, sumado a que contribuye a la presencia de especies r (Ritter et al. 2005, Egilsdottir et al. 2009, Sawada et al. 2011, Quintana et al. 2015).

A su vez, la evaluación de los procesos de reclutamiento ha permitido establecer que el cambio en la densidad de comunidades bentónicas ocurre por fluctuaciones en los valores de temperatura y salinidad, transporte debido a la corriente, morfología de zonas costeras y éxito en la sobrevivencia (Gaines \& Bertness 1992, Connolly et al. 2001, Strathmann et al. 2002). Por otra parte, la presencia de especies con múltiples generaciones al año (multivoltinas), puede generar un efecto marcado en la dinámica de las poblaciones y en el tamaño promedio de los organismos que las componen, por la aparición de individuos de menor talla en diferentes épocas del año $(<0,5 \mathrm{~mm})$ (Gillooly et al. 2001).

Por todo lo anteriormente expuesto, los propósitos de este trabajo son: a) probar la capacidad de retención de los tamices de 1,0 y $0,5 \mathrm{~mm}$, para calcular la densidad (ind $\mathrm{m}^{-2}$ ) y biomasa (g AFDW $\mathrm{m}^{-2}$ ), b) determinar el cambio de tamaño en los organismos de la comunidad bentónica asociados a una pradera de Zostera marina, ubicado en El Estero de Punta Banda a lo largo de dos años de estudio. 


\section{MATERIALES Y MÉTODOS}

\section{ÁREA DE ESTUdio Y DISEÑo DEL MUESTREO}

El Estero de Punta Banda (referido como Estero en adelante) ubicadoen el Estado de Baja California, México, es una laguna costera que presenta las características de antiestuario, la cual se encuentra ubicada al sureste de la Bahía de Todos Santos entre los $31^{\circ} 46^{\prime} 36^{\prime \prime}-31^{\circ} 41^{\prime} 40^{\prime \prime} \mathrm{N}$ y $116^{\circ} 39^{\prime} 26^{\prime \prime}$ $116^{\circ} 37^{\prime} 36^{\prime \prime} \mathrm{W}$ (Fig. 1). El Estero está separado de la bahía por una barra de arena de $7 \mathrm{~km}$, presenta una sola boca de $150 \mathrm{~m}$ de ancho en marea baja, un rango máximo de marea de 2,5 $\mathrm{m}$ y una marea promedio de 1,04 $\mathrm{m}$ con corrientes de mareas de $5 \mathrm{~cm} \mathrm{~s}^{-1}$ (Acosta-Ruiz \& Álvarez-Borrego 1974, Pritchard et al. 1978).

El muestreo se realizó en una pradera de Zostera marina ubicada a $31^{\circ} 46^{\prime}-31^{\circ} 41^{\prime} \mathrm{N}$ y $116^{\circ} 39^{\prime}-116^{\circ} 37^{\prime} \mathrm{W}$ (Fig. 1), durante la marea más baja, procurando mantener una frecuencia bimestral desde el 7 de agosto 2009 hasta el 1 de mayo 2011. En cada fecha de muestreo, se colectaron 4 muestras de sedimento (núcleos) utilizando un nucleador área de $176,8 \mathrm{~cm}^{2}$. Cada núcleo fue fijado con una solución de formalina al $7 \%$, neutralizada con borato de sodio.

\section{Procesamiento del material biológico}

El sedimento fue tamizado en húmedo y el material biológico separado usando una serie de tamices de 1,0, 0,5 y 0,35 mm de diámetro de malla. Los organismos retenidos en el tamiz de 0,35 mm se consideraron como el $100 \%$ de retención. La fauna retenida en cada tamiz se separó con la ayuda de un microscopio estereoscopio. Los organismos fueron contados e identificados al nivel taxonómico de clase, excepto los nemátodos los cuales se dejaron al nivel de phyllum.

El peso húmedo de todo el material biológico se midió con una balanza de precisión Denver Instrument M220D con resolución de $0,01 \mathrm{~g}$, los valores obtenidos fueron corregidos utilizando el factor de 1,2 aportado por Brey (1986) para evitar la pérdida de peso asociada a la fijación y preservación de los organismos con formol y etanol. El peso seco libre de cenizas (g AFDW) fue estimado haciendo uso de los factores de conversión proporcionados por Brey (1990) quien proporciona valores específicos para cada taxa. Todos los organismos fueron identificados a nivel de clase y preservados en etanol al $70 \%$ para el posterior análisis taxonómico.

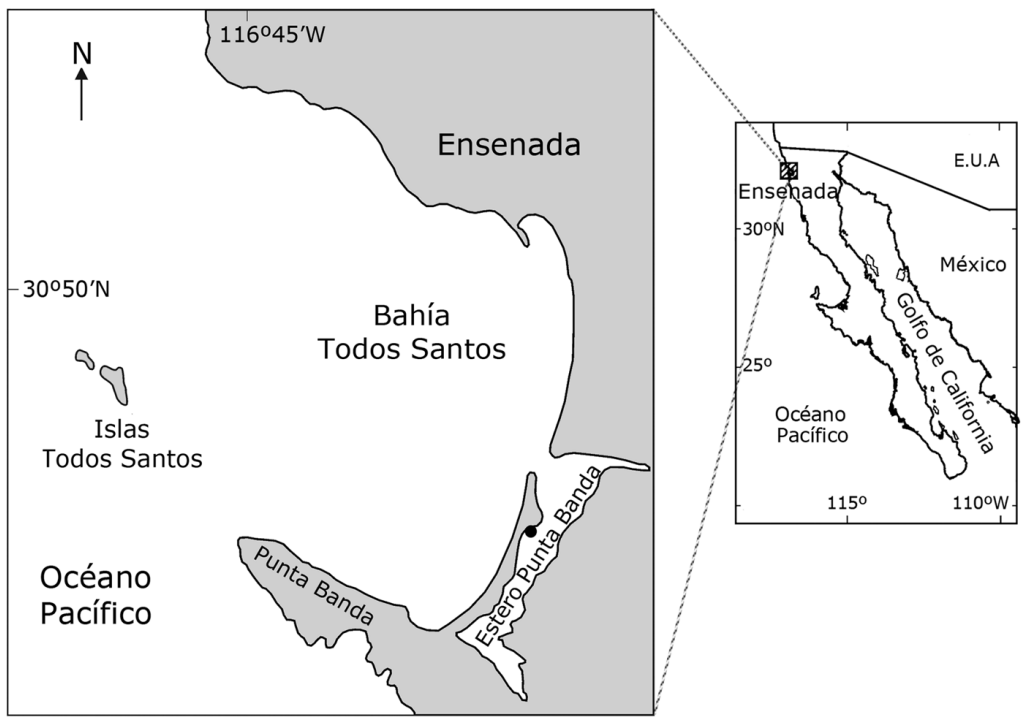

Figura 1. Estero de Punta Banda ubicado en la costa del Pacifico mexicano cerca de la ciudad de Ensenada, Baja California, México. El punto negro indica el área de muestro / Estero de Punta Banda located in the Pacific mexican coast near to Ensenada city, Baja California, México. The black point indicates the sampling site 


\section{Tratamiento estadístico}

Con el fin de representar ciclos anuales en los cuales es posible observar variaciones debidas a factores ambientales (e.g., estacionalidad), biológicos (e.g., reclutamiento) y comparar los resultados del presente estudio con los aportados en aquellos realizados en diferentes áreas por espacio de un año; los datos de densidad y biomasa por metro cuadrado fueron agrupados en 2 períodos. El primer período comprende los muestreos de agosto 2009 a abril 2010, mientras que el segundo período comprende los realizados entre julio 2010 a mayo 2011. Todos los valores de abundancia y biomasa promedio fueron acompañados con sus respectivos errores estándar. Los porcentajes de retención de los tamices de 1,0 mm y 0,5 mm se calcularon asumiendo como $100 \%$ los valores de abundancia y biomasa obtenidos en el tamiz de 0,35 mm, además se calculó la cantidad porcentual de pérdida de organismos por el uso del tamiz de 1,0 mm (pérdida se refiere a todos los organismos no retenidos en el tamiz). Para homogeneizar las varianza, los datos de densidad y biomasa fueron transformados utilizando raíz cuarta (Downing 1979, Clarke 1993, Hernández-Arana et al. 2005). La retención de organismos de los tamices de 1,0 y 0,5 mm fue comparada con la prueba t-pareada dado que las unidades experimentales son dependientes y cumplen como los supuestos de las pruebas paramétricas. Para establecer cambios en el tamaño en los organismos por taxa entre los 2 períodos, se compararon los valores de retención de biomasa y densidad en los tamices de 1,0 y $0,5 \mathrm{~mm}$, con la prueba no paramétrica de Mann-Whitney. Todas las pruebas estadísticas fueron realizadas en el programa BioEstat 2.0.

\section{Resultados}

\section{DENSIDAD}

Durante los 2 períodos fueron colectados un total de 13.606 individuos pertenecientes a 9 phyla y 10 clases. La clase Malacostraca agrupó a los anfípodos, isópodos, decápodos y cumáceos, siendo los anfípodos e isópodos los crustáceos más abundantes en los 2 períodos. La densidad promedio total del primer período fue de $110.817 \pm 47.981$ ind $\mathrm{m}^{-2}$, mientras que la del segundo fue $104.949 \pm 40.021$ ind $\mathrm{m}^{-2}$.

En los 2 períodos se observaron fluctuaciones en la densidad y tamaño de los organismos retenidos en los 2 tamices, lo cual fue evidente en la disminución de la densidad entre los meses de verano a invierno; en los meses de primavera los valores tendieron a aumentar (Fig. 2). El primer período presentó los 2 valores extremos de todo el estudio, en enero se presentó el valor más bajo de densidad promedio por fecha de muestreo $\left(1824 \pm 1039\right.$ ind $\left.\mathrm{m}^{-2}\right)$ y en agosto se observó el valor más elevado $\left(45.149 \pm 21.469\right.$ ind $\left.\mathrm{m}^{-2}\right)$, mientras que en el segundo período los valores fluctuaron entre $13.249 \pm 3709$ ind $\mathrm{m}^{-2}$ en enero y $25.509 \pm 15.574$ ind $\mathrm{m}^{-2}$ en julio (Fig. 2).

El patrón de retención de organismos fue similar en los 2 períodos, ya que el tamiz de $0,5 \mathrm{~mm}$ presentó el mayor número de organismos con respecto al de 1,0 mm (Fig. 2). Debido al uso del tamiz de $1,0 \mathrm{~mm}$ se perdió un $44 \%$ del total de organismos presentes en las muestras durante el primer período y de $39 \%$ en el segundo; la pérdida de organismos pertenecientes a la macrofauna fue de $35 \%$ en el primer período, mientras que en el segundo período fue del $41 \%$, mientras que la pérdida de poliquetos fluctuó entre 34 al $36 \%$ en el primer y segundo período, respectivamente (Fig. 3).

La retención de organismos de los tamices de 1,0 y $0,5 \mathrm{~mm}$ fue significativamente diferente en los 2 períodos ( $\mathrm{t}$ pareada $=$ $-2,76$, g.l. $=12, P=0,01 ; \mathrm{t}$ pareada $=-2,46, \mathrm{~g} .1 .=12, P=0,02$, respectivamente).

\section{Biomasa}

La biomasa promedio total de los 2 períodos fue de 38,21 \pm $26,27 \mathrm{~g} \mathrm{AFDW} \mathrm{m}^{-2}$. En el primer período la biomasa promedio total fue mayor que en el segundo $(25,25 \pm 21,33$ y $12,96 \pm$ $12,90 \mathrm{~g} \mathrm{AFDW} \mathrm{m}^{-2}$, respectivamente), en los 2 períodos los poliquetos retenidos en el tamiz de $1,0 \mathrm{~mm}$ presentaron los valores de biomasa más altos, su aporte fue de 16,06 $\pm 7,15 \mathrm{~g}$ AFDW m ${ }^{-2}$ en el primer período y 5,66 $\pm 2,41 \mathrm{~g} \mathrm{AFDW} \mathrm{m}^{-2}$ en el segundo (Fig. 4). La biomasa de los moluscos fluctuó entre $4,23 \pm 6,63 \mathrm{~g} \mathrm{AFDW} \mathrm{m}^{-2}$ en el primer período y 2,58 $\pm 1,44 \mathrm{~g}$ AFDW $\mathrm{m}^{-2}$ en el segundo; mientras que los valores de los crustáceos variaron entre $3,26 \pm 2,48 \mathrm{~g} \mathrm{AFDW} \mathrm{m}^{-2}$ en el primer período y 4,25 $\pm 4,07 \mathrm{~g}$ AFDW m ${ }^{-2}$ en el segundo. Los demás phyla presentaron valores de biomasa menores a 0,5 g AFDW $\mathrm{m}^{-2}$ o por debajo de nuestro nivel de detección (Fig. 4).

En ambos períodos la biomasa presentó porcentajes de retención similares en los tamices de $1,0 \mathrm{~mm}$ y 0,5 mm (87 y 97\%, respectivamente) (Fig. 4), lo cual se refleja en la ausencia de diferencias significativas en la retención de los 2 tamices en los 2 períodos ( $\mathrm{t}$ pareada $=-1,22$, g.l $=10, P=0,24$; $\mathrm{t}$ pareada $=$ $-1,82$, g.l. $=7, P=0,11)$.

\section{TAlla De ORGANismos}

Los valores de biomasa y abundancia por taxa entre períodos no presentaron diferencias significativas $(P>0,05)$ (Tabla 1$)$, lo cual indica que el tamaño de los organismos de la comunidad no presentó cambios significativos. 


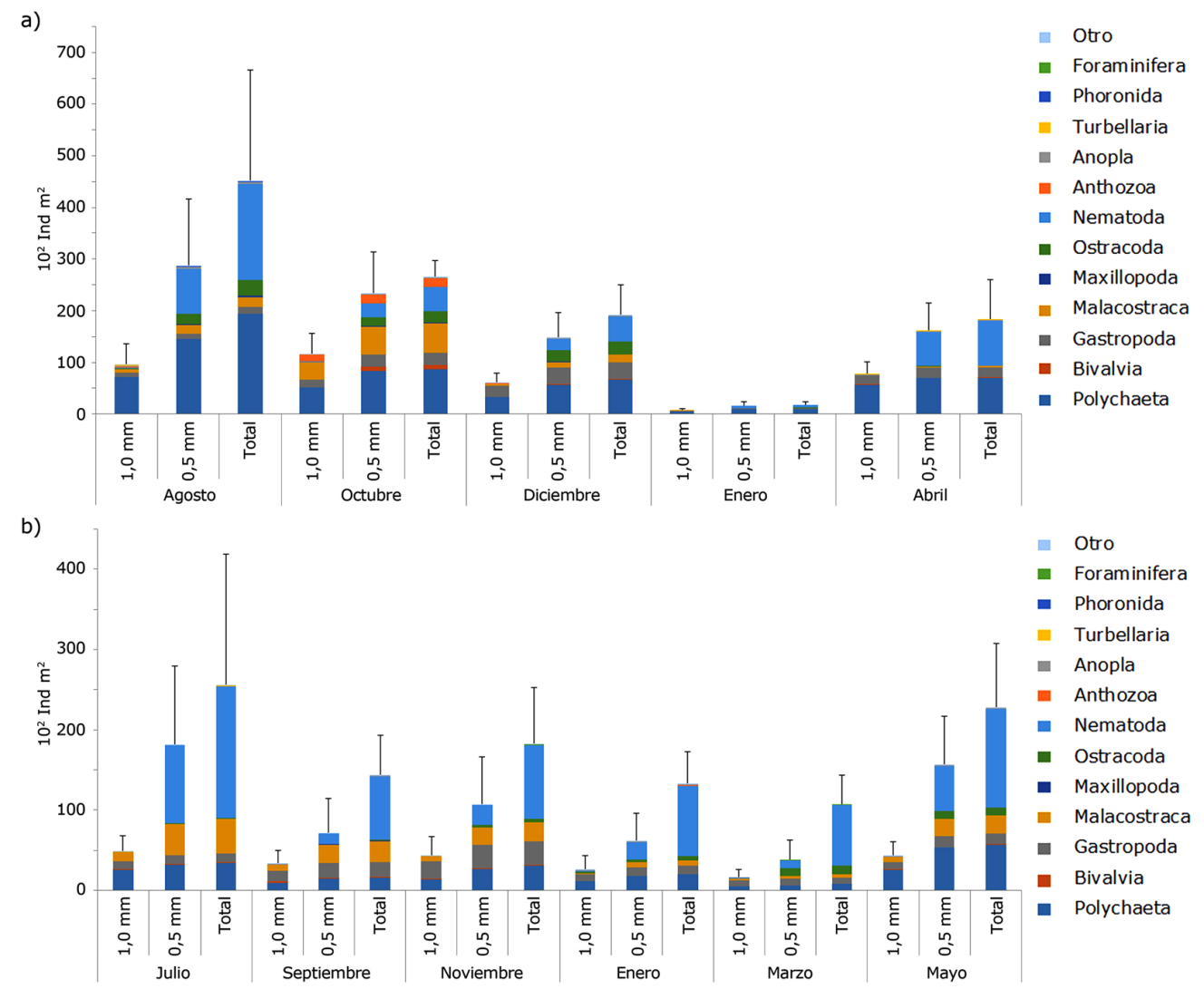

Figura 2. Densidad de organismos por metro cuadrado, por mes de muestreo y diámetro de malla usado ( $\pm \mathrm{E}$.E); los valores en $10^{2}$ ind $\mathrm{m}^{-2}$, a) primer período, b) segundo período / Density of organisms per meter square by month of sampling and mesh size used ( \pm S.E); values are in $10^{2}$ ind $\mathrm{m}^{-2}$, a) first period, b) second period

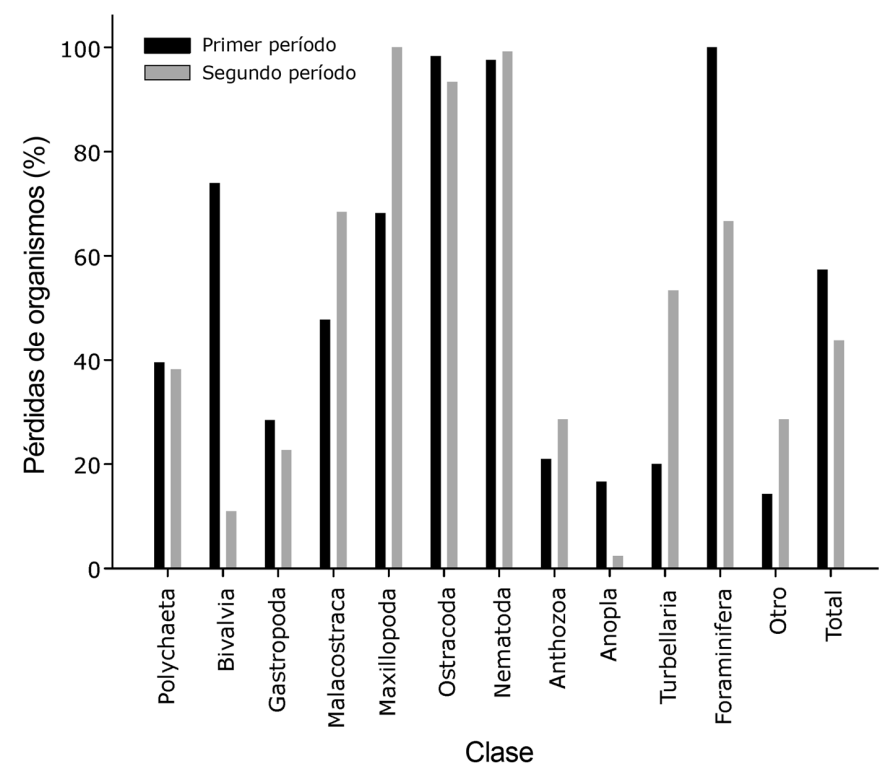

Figura 3. Porcentaje de pérdida de organismos debido al uso del tamiz de $1,0 \mathrm{~mm} /$ Percentage of organisms loss due to use of $1.0 \mathrm{~mm}$ sieve 

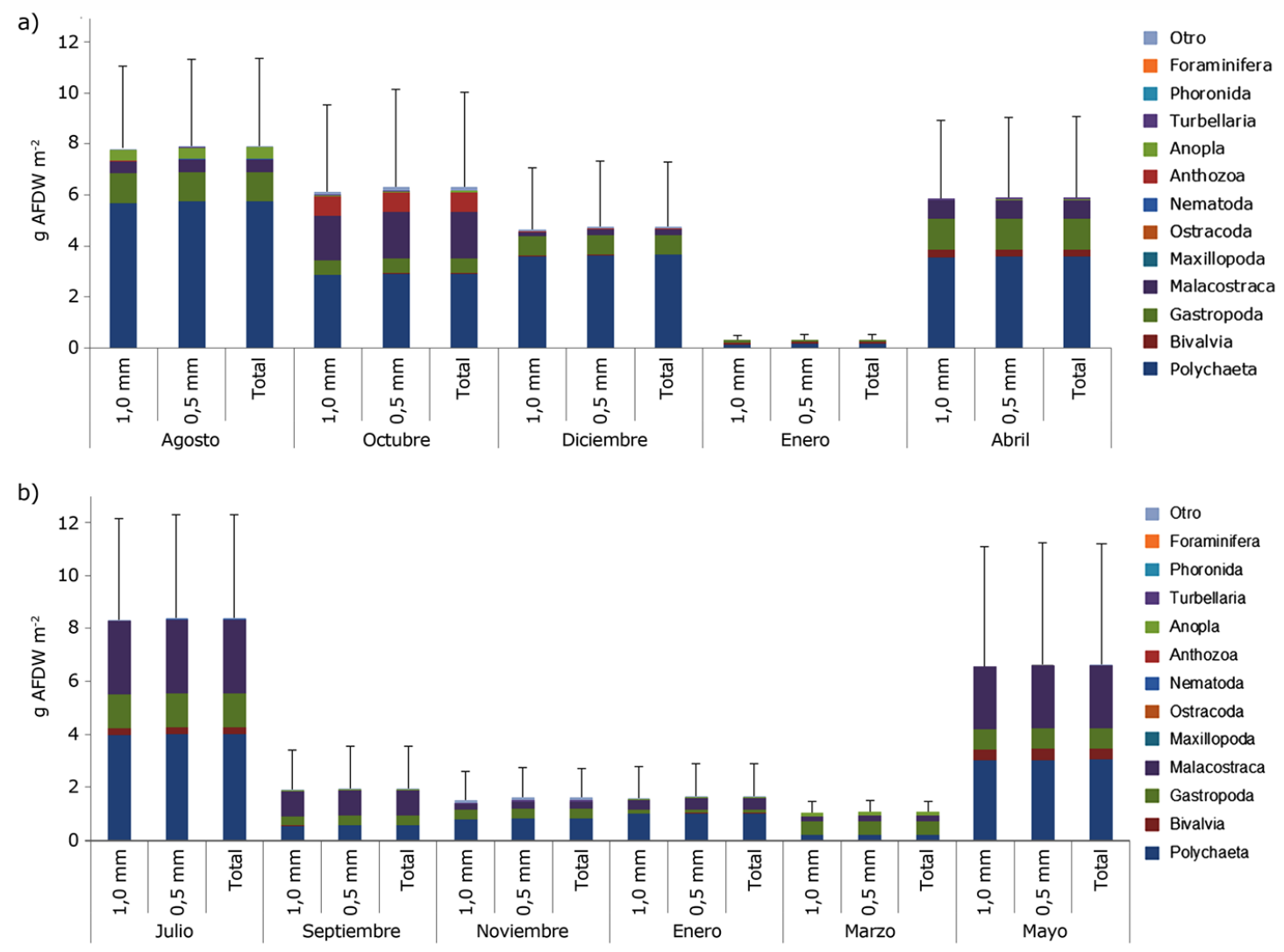

Figura 4. Biomasa por metro cuadrado, por mes de muestreo y diámetro de malla ( \pm E.E). Los valores en gramos de peso seco libre de cenizas por sus siglas en ingles $\mathrm{AFDW} \mathrm{\textrm {m } ^ { - 2 }}$ a) primer período, b) segundo período / Biomass per meter square by month of sampling and mesh size used ( \pm S.E). The values are in grams of ash free dry weight by acronym in English g AFDW ${ }^{-2}$ a) first period, b) second period

\section{DiscuSIón}

\section{DENSIDAD}

La densidad de organismos en el área de estudio se encuentra influida por la presencia de nemátodos, lo cual concuerda con los datos reportados por Solana et al. (2014) para la zona de estudio durante un período de muestreo anterior al del presente estudio. Sin embargo, Talley et al. (2000) al evaluar la comunidad infaunal al otro lado del canal central, no reporta dichos organismos a pesar de haber usado el tamiz de $0,3 \mathrm{~mm}$; indicando que la distribución de los nemátodos al igual que la exhibida por los poliquetos de la familia Capitellidae, siguen una distribución espacial irregular o agregada debida a variaciones ambientales del Estero.

De aquí que la fluctuación estacional típica de áreas templadas fue evidente en los valores de densidad del área de estudio debido a la presencia de numerosos poliquetos de tamaño pequeño de las familias Nereididae, Orbiniidae,

Spionidae y Sabellidae, el aumento en la abundancia de crustáceos, así como a la presencia de huevos de moluscos en primavera y verano (Fig. 2). Dicho patrón fue reportado por Calderón-Aguilera (1992) en la Bahía de San Quintín, quien lo atribuye al reclutamiento y reproducción de los miembros de la comunidad bentónica, los cuales aumentan la densidad del área evaluada. A su vez, los reclutas sirven de alimento a otros organismos y son distribuidos en otras áreas como producto de la fluctuación de la comunidad debido a la hidrodinámica propia del Estero (Underwood \& Fairweather 1989, Hughes et al. 2007).

Estos resultados muestran claramente que la densidad de organismos siguen el patrón unimodal de áreas templadas observado en diferentes ecosistemas estuarinos (Heck \& Orth 1980, Quiroz-Vázquez 2005), el cual es más evidente al utilizar 
Tabla 1. Valores de la prueba de Mann-Whitney para determinar diferencias en el tamaño de los organismos entre los dos períodos / Values of Mann-Whitney test to determine differences in the organisms' size between both periods

\begin{tabular}{|c|c|c|c|c|c|c|}
\hline & \multicolumn{3}{|c|}{$1,0 \mathrm{~mm}$} & \multicolumn{3}{|c|}{$0,5 \mathrm{~mm}$} \\
\hline & $\mathrm{U}$ & $Z(U)$ & $P$ & $\mathrm{U}$ & $\mathrm{Z}(\mathrm{U})$ & $P$ \\
\hline \multicolumn{7}{|l|}{ Densidad } \\
\hline Polychaeta & 5 & 1,82 & 0,06 & 5 & 1,82 & 0,06 \\
\hline Bivalvia & 14,5 & 0,09 & 0,92 & 11 & 0,73 & 0,46 \\
\hline Gastropoda & 12 & 0,54 & 0,58 & 11 & 0,73 & 0,46 \\
\hline Malacostraca & 10,5 & 0,82 & 0,41 & 10 & 0,91 & 0,36 \\
\hline Maxillopoda & 12 & 0,54 & 0,58 & 8 & 1,27 & 0,20 \\
\hline Ostracoda & 12,5 & 0,45 & 0,64 & 10,5 & 0,82 & 0,41 \\
\hline Nematoda & 9 & 1,09 & 0,27 & 13,5 & 0,27 & 0,78 \\
\hline Anthozoa & 9 & 1,09 & 0,27 & 9,5 & 1,00 & 0,31 \\
\hline Anopla & 12 & 0,54 & 0,58 & 10 & 0,91 & 0,36 \\
\hline Turbellaria & 12,5 & 0,45 & 0,64 & 14 & 0,18 & 0,85 \\
\hline Phoronida & 14 & 0,18 & 0,85 & 14 & 0,18 & 0,85 \\
\hline Foraminifera & 12,5 & 0,45 & 0,64 & 13 & 0,36 & 0,71 \\
\hline Otro & 14 & 0,18 & 0,85 & 13,5 & 0,27 & 0,78 \\
\hline \multicolumn{7}{|l|}{ Biomasa } \\
\hline Annelida & 10 & 0,91 & 0,36 & 10 & 0,91 & 0,36 \\
\hline Mollusca & 7 & 1,46 & 0,14 & 7 & 1,46 & 0,14 \\
\hline Arthropoda & 11 & 0,73 & 0,46 & 11 & 0,73 & 0,46 \\
\hline Cnidaria & 8 & 1,27 & 0,20 & 8 & 1,27 & 0,20 \\
\hline Nemertea & 8,5 & 1,18 & 0,23 & 8,5 & 1,18 & 0,23 \\
\hline Nematoda & 14 & 0,18 & 0,85 & 13,5 & 0,27 & 0,78 \\
\hline Sipuncula & 12,5 & 0,45 & 0,64 & 10 & 0,91 & 0,36 \\
\hline Huevos & 15 & 0,0 & 1,0 & 15 & 0,0 & 1,0 \\
\hline Otro & 12,5 & 0,45 & 0,64 & 12,5 & 0,45 & 0,64 \\
\hline
\end{tabular}

el tamiz de 0,5 mm (Bachelet 1990). Esto, además permite observar el aumento en la densidad de organismos debido a reclutamiento, ocurrido durante agosto, octubre y mayo; dicho reclutamiento estuvo altamente marcado por poliquetos y nematodos en agosto y julio respectivamente, mientras que los crustáceos y gasterópodos lo presentaron en julio y noviembre en el segundo periodo (Fig. 2).

Posiblemente factores ambientales como tormentas o inundaciones están afectado la estructura poblacional (Gaines \& Bertness 1992), ya que en el área se observó la remoción de la capa superficial del sedimento del área; indicando que dichos eventos debieron presentar velocidades mayores a 5 $\mathrm{cm} \mathrm{s}^{-1}$, la cual corresponde a la velocidad promedio del Estero (Pritchard et al. 1978). Dolbeth et al. (2007) y Grilo et al. (2011) han reportado disminuciones en las densidades de comunidades bentónicas debido a dichos factores ambientales, los cuales afecta en mayor medida a los organismos macrofaunales (Schwinghamer 1983), razón por la cual es posible atribuir a estos eventos físicos la disminución de organismos en el área evaluada (Fig. 2). Sin embargo, para determinar el efecto de las variables responsables de la hidrodinámica y recambio de aguas del Estero sobre la fauna bentónica presente en el Estero, se deben diseñar experimentos con una mayor frecuencia de muestreo a la del presente estudio.

Los porcentajes de pérdida de organismos de este estudio fueron similares a los aportados otras investigaciones (Schlacher \& Wooldridge 1996, Tanaka \& Pereira 1998, Gage et al. 2002, Lampadariou et al. 2005), ratificando que el tamiz de $1,0 \mathrm{~mm}$ es una herramienta con la que debiera esperarse un porcentaje de perdida alrededor del 40 al $82 \%$ para ambientes estuarinos.

Aunque el tiempo de separación de organismos se reduce al utilizar el tamiz de $1,0 \mathrm{~mm}$, dando a su vez la posibilidad de procesar mayor número de réplicas (Couto et al. 2010, Pinna et al. 2013), muchas especies que son moderadamente abundantes pueden ser escasas en el material colectado; situación que es conspicua al usar el diámetro de malla de 1,0 mm (Schlacher \& Wooldridge 1996). Esto origina subestimaciones debidas a la elección de un diámetro de malla mayor a 0,5 mm (Schlacher \& Wooldridge 1996, Gage et al. 2002).

Por lo anterior, es recomendable usar un tamiz de menor diámetro de malla, con el fin de establecer los parámetros comunitarios y ecológicos que dependen de la presenciaausencia, riqueza, densidad de especies y reclutamiento (Bachelet 1990, Abel 1996, Rodrigues et al. 2007, Barba et al. 2010); según lo expresado por Morin et al. (2004) y Barba et al. (2010) se detectan adecuadamente utilizando el tamiz de $0,5 \mathrm{~mm}$ (Fig. 2). Esto es porque los organismos de talla pequeña no son excluidos, lo cual es evidente en las diferencias significativas observada entre los tamices de 1,0 y $0,5 \mathrm{~mm}$ del presente estudio.

Teniendo en cuenta los resultados aportados por el presente estudio y las evidencias aportadas por otros autores, es recomendable utilizar el tamiz de $0,5 \mathrm{~mm}$ con el fin de tener datos robustos y mantener el tamaño mínimo de muestra por estación de muestreo recomendado por Holme \& McIntyre (1984) que corresponde a $0,1 \mathrm{~m}^{2}$.

\section{Biomasa}

La carencia de diferencias significativas entre los tamices de 1,0 y $0,5 \mathrm{~mm}$ en los 2 períodos, ha sido observado en comunidades bentónicas de ríos, estuarios y fondos blandos marinos (Bachelet 1990, Morin et al. 2004, Lampadariou et al. 2005). Para la biomasa el diámetro de malla no aporta sesgos sobre la información obtenida. Esto pudo ser resultado de la 
baja variabilidad interanual de los valores de biomasa de macroinvertebrados (casi constantes) reportado por Beukema et al. (1978), aunado a la propiedad conservativa de los valores de biodiversidad (Schwinghamer 1983) y la presencia de organismos de estrategia $r$ que prosperan en ambientes que han sufrido algún tipo de perturbación (Boesch \& Point 1976).

A su vez en el presente estudio, los poliquetos fueron el grupo taxonómico responsable en mayor medida de los valores de biomasa del área evaluada, lo cual contrasta con lo reportado por Schlacher \& Wooldridge (1996) y QuirozVasquez et al. (2005) quienes documentan que los crustáceos peracáridos representaron el mayor porcentaje de biomasa (66 y $36,5 \%$, respectivamente), mientras que la biomasa de los poliquetos representaron el 31 y $0,2 \%$, respectivamente. Pese a la variabilidad taxonómica observada entre estudios la retención de organismos por parte de los 2 tamices y su contribución a los valores de biomasa es lo suficientemente confiable para evaluar dicha variable.

\section{Talla de ORGanismos}

Los valores de densidad y biomasa de los organismos retenidos en un tamiz en particular presentan un sesgo asociado a la presencia de desecho vegetal, en el cual organismos de menor tamaño quedan retenidos en tamices de diámetro de malla mucho mayor al que corresponden (Morin et al. 2004); sumado a esto, organismos elongados (e.g., poliquetos) pueden quedar retenidos en tamices de menor diámetro al que corresponden, debido a que pueden pasar a través de la malla del tamiz (Morin et al. 2004), situación que pudo influir los resultados obtenidos en este y otros estudios.

Teniendo en cuenta que la biomasa sea una medida del volumen (Crisp 1984), se podría determinar que esta es la mejor variable para estimar el tamaño de los miembros de la comunidad, sin embargo los resultados obtenidos en este estudio no permiten llegar a esa conclusión, ya que se evaluó dicho objetivo teniendo en cuenta tanto la densidad como la biomasa de los organismos retenidos en los tamices evaluados.

La ausencia de diferencias significativas en los valores de densidad y biomasa por taxa entre los tamices de 1,0 y $0,5 \mathrm{~mm}$ (Tabla 1), indican que la cantidad de organismos retenida en los 2 períodos fue similar, lo cual contrasta con lo reportado por Solana-Arellano et al. (2014) quienes determinaron en un período previo (2008-2009) para el área evaluada, que los tamices de 1,0 y 0,5 mm fueron los adecuados para valorar de la densidad de la comunidad; indicando una disminución en la talla de los organismos entre los 2 estudios. Esto puede ser atribuido a la ausencia del efecto perturbador ocasionado por el transporte de sedimento realizado por las tormentas, inundaciones e incidencia del viento sobre la pradera durante el estudio de Solana-Arellano et al. (2014).

Dado que las perturbaciones favorecen a los grupos taxonómicos de estrategia $r$, los cuales se caracterizan por presentar tamaño pequeño y altas tasas de crecimiento y reproducción, que les permite mantenerse en el área afectada durante el evento y recuperarse rápidamente después que este haya sucedido (Boesch \& Point 1976, Posey \& Alphin 2002, Arntz et al. 2006); puede que sea su presencia, sumado a la baja estabilidad del ambiente (debido a la variabilidad climática del área de estudio), los responsables de la ausencia de diferencias en el tamaño de los miembros de la comunidad evaluada.

En relación al reclutamiento de los miembros de la comunidad, presumimos que continuó con el patrón unimodal de áreas templadas (Quiroz-Vázquez 2005), ya que cambios significativos debido a las perturbaciones climáticas o no medidas en el presente estudio, habrían sido detectadas en la comparación de tamaño entre periodos (de manera más clara en términos de biomasa), por diferencias en el tiempo de desarrollo larval, asentamiento y de incorporación a la comunidad (Ritter et al. 2005, Hughes et al. 2007, Sawada et al. 2011).

Teniendo en cuenta que los patrones ecológicos observables en comunidades bentónicas se ven afectados por la exclusión de organismos de talla pequeña y que dichos patrones se logran establecer de manera confiable usando el tamiz de $0,5 \mathrm{~mm}$, según el presente estudio, se recomienda el uso de dicho diámetro de malla con el objetivo de aportar datos robustos a las evaluaciones realizadas sobre comunidades bentónicas, permitiendo determinar con mayor precisión la variabilidad espaciotemporal.

\section{Agradecimientos}

J.C. Rubio-Polania agradece a Jagganath y al Consejo Nacional de Ciencia y Tecnología de México (CONACYT) a través del programa de becas de posgrado por su apoyo. Los autores agradecemos a Martin Serrano-Tadeo por su trabajo en laboratorio, Francisco Ponce por la edición de las figuras, Eliana Gómez-Ocampo, Manuel Mariano-Matías, Leilani Meandros, Nadia Siordia, Geovana Leon por su apoyo logístico en la fase final del manuscrito. A los evaluadores anónimos agradecemos la contribución realizada a través de sus comentarios que permitieron mejorar la calidad del documento. 


\section{LITERATURA CITADA}

Abel PD. 1996. Water pollution biology, 268 pp. Taylor \& Francis, London.

Acosta-Ruiz M \& S Álvarez-Borrego. 1974. Distribución superficial de algunos parámetros hidrológicos físicos y químicos, en el Estero de Punta Banda, B. C., en otoño e invierno. Ciencias Marinas 1(1): 16-45.

Arntz WE, V Gallardo, D Gutiérrez, E Isla, L Levin, J Mendo, C Neira, GT Rowe \& M Wolff. 2006. El Niño and similar perturbation effects on the benthos of the Humboldt, California, and Benguela Current upwelling ecosystems. Advance in Geoscience 6: 243-265.

Bachelet G. 1990. The choice of a sieving mesh size in the quantitative assessment of marine macrobenthos: a necessary compromise between aims and constraints. Marine Environmental Research 30: 21-35.

Barba B, A Larra, A Otermin, A Basaguren \& J Pozo. 2010. The effect of sieve mesh size on the description of macroinvertebrate communities. Limnetica 29(2): 211-220.

Beukema J, W De Bruin \& J Jansen. 1978. Biomass and species richness of the macrobenthic animals living on the Tidal flats of the dutch wadden sea: long-term changes during a period With mild winters. Netherlands Journal Sea Research 12(1): 58-77.

Boesch F \& G Point. 1976. Effects of tropical storm agnes on soft-bottom macrobenthic communities of the James and York Estuaries and the Lower Chesapeake Bay. Chesapeake Science 17(4): 246-259.

Botsford LW. 2001. Physical influences on recruitment to California Current invertebrate populations on multiple scales. Journal of Marine Science 58: 1081-1091.

Brey BT. 1986. Formalin and Formaldehyde-depot chemicals: effects on dry weight and ash free dry weight of two marine bivalve species. Berichte der Deutschen Wissenschaftlichen Kommission fur Meeresforschung 31: 52-57.

Brey BT. 1990. Estimating productivity of macrobenthic invertebrates form biomass and mean individual weight. Berichte der Deutschen Wissenschaftlichen Kommission fur Meeresforschung 32: 329-343.

Buss D \& E Borges. 2008. Application of rapid bioassessment protocols (RBP) for benthic macroinvertebrates in Brazil: comparison between sampling techniques and mesh sizes. Neotropical Entomology 37(3): 288-295.

Calderón-Aguilera LE. 1992. Análisis de la infauna béntica de San Quintín, Baja California, con énfasis en su utilidad en la evaluación de impacto ambiental. Ciencias Marinas 18(4): $27-46$.

Clarke KR. 1993. Non-parametric multivariate analyses of changes in community structure. Australian Journal of Ecology 18: 117-143.

Connolly SR, B Menge \& J Roughgarden. 2001. A latitudinal gradient in recruitment of intertidal invertebrates in the northeast Pacific ocean. Ecology 82: 1799-1813.

Couto T, J Patrício, J Neto, F Ceia, J Franco \& JC Marques. 2010. The influence of mesh size in environmental quality assessment of estuarine macrobenthic communities. Ecological Indicators 10: 1162-1173.
Crisp D. 1984. Energy flow measurements. In: Holme N \& A McIntire (eds). Methods for the study of marine benthos, pp. 284-372. Blackwell Scientific Publication, Oxford.

Dolbeth M, Ó Ferreira, H Teixeira, JC Marques, JA Dias \& MA Pardal. 2007. Beach morphodynamic impact on a macrobenthic community along a subtidal depth gradient. Marine Ecology Progress Series 352: 113-124.

Downing JA. 1979. Aggregation, transformation and the desing of benthos sampling programs. Journal of Fisheries Research Board of Canada 36: 1454-1463.

Egilsdottir H, JI Spicer \& SD Rundle. 2009. The effect of $\mathrm{CO} 2$ acidified sea water and reduced salinity on aspects of the embryonic development of the amphipod Echinogammarus marinus (Leach). Marine Pollution Bulletin 58(8): 1187-1191.

Eleftheriou A \& A McIntyre. 2005. Methods for the study of marine benthos, $496 \mathrm{pp}$. Blackwell Science, Oxford.

Gage J, D Hughes \& J Gonzalez-Vecino. 2002. Sieve size influence in estimating biomass, abundance and diversity in samples of deep-sea macrobenthos. Marine Ecology Progress Series 225: 97-107.

Gaines SD \& MD Bertness. 1992. Dispersal of juveniles and variable recruitment in sessile marine species. Nature 360: 579-580.

Gillooly J, J Brown, G West, V Savage \& E Charnov. 2001. Effects of size and temperature on metabolic rate. Science 293: 2248-2252.

Gray J \& M Elliot. 2009. Ecology of sediments from science to management, $225 \mathrm{pp}$. Oxford University Press, Oxford.

Grilo TF, PG Cardoso, M Dolbeth, MD Bordalo \& M Pardal. 2011. Effects of extreme climate events on the macrobenthic communities' structure and functioning of a temperate estuary. Marine Pollution Bulletin 62(2):303-311.

Heck KL \& RJ Orth. 1980. Structural components of edarckelgrass (Zostera marina) meadows in the lower Chesapeake. Estuaries 3: 289-295.

Hernández-Arana H, R Warwick, M Attrill, A Rowden \& G Gold-Bouchot. 2005. Assessing the impact of oil-related activities on benthic macroinfauna assemblages of the Campeche shelf, southern Gulf of Mexico. Marine Ecology Progress Series 289: 89-107.

Holme N \& A McIntyre. 1971. Methods for the study of marine benthos. IBP Handbook 16: 1-332. Blackwell Scientific Publications, Oxford.

Hughes TP, AH Baird, EA Dinsdale, NA Moltschaniwskyj, MS Pratchett, JE Tanner \& BL Willis. 2007. Supplyside ecology works both ways: the link between benthic adults, fecundity, and larval recruits. Ecology 81:2241-2249.

Lampadariou N, I Karakassis \& TH Pearson. 2005. Cost/ benefit analysis of a benthic monitoring programme of organic benthic enrichment using different sampling and analysis methods. Marine Pollution Bulletin 50(12): 1606-1618.

Largier J, J Hollibaugh \& S Smith. 1997. Seasonally hypersaline estuaries in Mediterranean-climate regions. Estuaries and Coastal Shelf Science 45: 789-797.

Morin A, J Stephenson, J Strike \& A Solimini. 2004. Sieve retention probabilities of stream benthic invertebrates. Journal of the North American Benthological Society 23(2): 383-391. 
Pearson T \& R Rosenberg. 1978. Macrobenthic succession in relation to organic enrichment and pollution of the marine environment. Oceanography and Marine Biology: An Annual Review 16: 229-311.

Pinna M, G Marini, I Rosati, JM Neto, J Patrício, JC Marques \& A Basset. 2013. The usefulness of large bodysize macroinvertebrates in the rapid ecological assessment of Mediterranean lagoons. Ecological Indicators 29: 48-61.

Pocklington P \& PG Wells. 1992. Key taxa for marine environmental monitoring. Marine Pollution Bulletin 24(12): 593-598.

Posey M \& T Alphin. 2002. Resilience and stability in an offshore benthic community: Responses to sediment borrow activities and hurricane disturbance. Journal of Coastal Research 18: 685-697.

Pritchard DW, R De la Paz Vela, H Cabrera-Muro, S Farreras-Sanz \& E Morales. 1978. Hidrografía física del Estero de Punta Banda. Parte I: Análisis de datos. Ciencias Marinas 5(2):1-23.

Quintana C, A Bernardino, P de Moraes, T Valdemarsen \& P Sumida. 2015. Effects of coastal upwelling on the structure of macrofaunal communities in SE Brazil. Journal of Marine Systems 143: 120-129.

Quiroz-Vásquez P, S Ibarra-Obando \& AE Meling-Lopez. 2005. Composition of the epifaunal community associated with the seagrass Zostera marina in San Quintin, Baja California. Bulletin of the Southern California Academy of Sciences 104(2): 100-112.

Reish D. 1959. A discussion of the importance of the screen size in washing quantitative marine bottom samples. Ecology 40(2): 307-309.

Ritter C, PA Montagna \& S Applebaum. 2005. Short-term succession dynamics of macrobenthos in a salinity-stressed estuary. Journal of Experimental Marine Biology and Ecology 323(1): 57-69.

Rodrigues A, S Meireles, T Pereira \& V Quintino. 2007. Spatial heterogeneity recognition in estuarine intertidal benthic macrofaunal communities: influence of sieve mesh-size and sampling depth. Hydrobiologia 587: 37-50.

Rosenberg R, M Blomqvist, H Nilsson, H Cederwall \& A Dimming. 2004. Marine quality assessment by use of benthic species-abundance distributions: a proposed new protocol within the European union water framework directive. Marine Pollution Bulletin 49: 728-739.

Rubio-Polania JC. 2013. Producción secundaria de la comunidad bentónica asociada a Zostera marina en el Estero de Punta Banda, Ensenada, Baja California, México. Tesis de Maestría, Departamento de Ecología Marina, CICESE. Ensenada, $81 \mathrm{pp}$.

Rygg B. 1985. Effect of sediment copper on benthic fauna. Marine Ecology Progress Series 25: 83-89.
Sawada H, H Saito, K Adachi \& H Toyohara. 2011. Seasonal variation of bivalve larvae on an exposed sandy beach on Kashima-nada: tips for the sandy beach recruitment process. Journal of Sea Research 65(2): 275-283.

Schlacher T \& TH Wooldridge. 1996. How sieve mesh size affects sample estimates of estuarine benthic macrofauna. Journal of Experimental Marine Biology and Ecology 201: 159-171.

Schwinghamer P. 1981. Characteristic size distributions of integral benthic communities. Canadian Journal Fish Aquatic Science 38: 1255-1263.

Schwinghamer P. 1983. Generating ecological hypotheses from biomass spectra using causal analysis: a benthic example. Marine Ecology Progress Series 13: 151-166.

Sellanes J, E Quiroga, C Neira \& D Gutiérrez. 2007. Changes of macrobenthos composition under different ENSO cycle conditions on the continental shelf off central Chile. Continental Shelf Research 27: 1002-1016.

Solana-Arellano E, V Díaz-Castañeda, O Flores-Uzeta, H Echavarría-Heras \& JC Rubio-Polanía. 2014. Assessment of secondary production and efficiency of different mesh sizes to study benthic communities associated to a Zostera marina meadow. Annual Research Review in Biology 4(16): 26042616.

Strathmann RR, TP Hughes, AM Kuris, KC Lindeman, SG Morgan, JM Pandolfi \& FJ Warner. 2002. Evolution of local recruitment and its consequences for marine populations. Bulletin of Marine Science 70: 377-396.

Talley T, P Dayton \& S Ibarra-Obando. 2000. Tidal flat macrofaunal communities and their associated environments in estuaries of southern California and northern Baja California, Mexico. Estuaries 23(1): 97-114.

Tanaka M \& FP Pereira. 1998. The effect of sieve mesh size on the abundance and composition of macrophyte-associated macrofaunal assemblages. Hydrobiologia 389: 21-28.

Thompson BW, MJ Riddle \& JS Stark. 2003. Cost-efficient methods for marine pollution monitoring at Casey Station, East Antarctica: the choice of sieve mesh-size and taxonomic resolution. Marine Pollution Bulletin 46: 232-243.

Underwood AJ \& PG Fairweather. 1989. Supply-side ecology and benthic marine assemblages. Trends in Ecology and Evolution 4: 16-20.

Warwick RM. 1993. Environmental impact studies on marine communities: Pragmatical considerations. Australian Journal of Ecology 18: 63-80.

Whitlow WL \& JH Grabowski. 2012. Examining how landscapes influence benthic community assemblages in seagrass and mudflat habitats in southern Maine. Journal of Experimental Marine Biology and Ecology 411: 1-6. 